\title{
The CHAllenges OF TREATing A PATIENT With RECURRENT CONGENITAL TOXOPLASMIC CHORIORETINITIS
}

\author{
Aleksandra Krasińska ${ }^{1}$, Kamila Jaz ${ }^{2 *}$, Joanna Mamczur ${ }^{2 *}$, Jarosław Kocięcki ${ }^{1}$
}

\begin{abstract}
Congenital toxoplasmosis is a rare, non-curable parasite infection, that affects approximately 242 children in Europe each year. Poland has one of the highest rates of congenital toxoplasmosis in Europe. Transmission of Toxoplasma gondii to the fetus results in numerous medical conditions, such as developmental delay, intellectual disabilities, seizures, hearing loss, and blindness. Chorioretinitis is a serious manifestation of congenital toxoplasmosis that can recur even after 25 years from the primary infection, which poses a significant therapeutic challenge. A 41-year-old female reported to the Ophthalmology Emergency Room due to blurred vision and pain in the right eye, accompanied by a constant headache. The patient suffered from congenital toxoplasmosis with two relapses in the past. On examination, the best-corrected visual acuity was 1,0 in both eyes, and the intraocular pressure was significantly increased. Slit-lamp examination showed vitritis and an active retinochoroidal lesion in the right eye. In the left eye, there was a retinochoroidal scar. A relapse of toxoplasmosis was suspected. Serology for Toxoplasma gondii was positive. Pyrimethamine with sulfadiazine, clindamycin, topical corticosteroids, and intraocular pressure-lowering drugs were implemented. During the treatment, the patient developed corticonuclear cataract in both eyes and reported psychotic symptoms. Clinical condition improved after the treatment with corticosteroids at a lower dose. Treatment of ocular manifestations of congenital toxoplasmosis is challenging. The clinical benefit of treatment should be weighed against side effects for each patient.
\end{abstract}

Running title: Congenital toxoplasmosis treatment

Keywords: congenital toxoplasmosis, chorioretinitis, retinochoroidal scar, uveitis

\footnotetext{
*equally contributed

${ }^{1}$ Department of Ophthalmology, Poznań University of Medical Sciences, Poznań, Poland

${ }^{2}$ Student of Poznań University of Medical Sciences, Poznań, Poland

*Correspondence: alex.krasinska@gmail.com

Full list of author information is available at the end of article
} 


\section{Introduction}

Congenital toxoplasmosis (CT) is a rare type of parasite infection that occurs when Toxoplasma gondii is transmitted to an infant during pregnancy [1]. The incidence of congenital toxoplasmosis varies depending on the region and is estimated at 6.7 per 100000 newborns in Europe. Poland has the second-highest incidence rate among the European countries. The serious consequences of acquiring infection from an early age and mortality rate of 2,5 $\%$ pose a major therapeutic challenge [2]. The treatment of ocular manifestations and central nervous system complications is difficult. In most cases, congenital ocular toxoplasmosis is primarily asymptomatic, however, untreated may lead to permanent vision loss. The aim of this paper is to present the treatment of a late relapse of congenital toxoplasmosis in an immunocompetent female.

\section{Case report}

A 41-year-old female reported to the Ophthalmology Emergency Room with blurred vision, pain in the right eye, and a constant headache. The patient was diagnosed with ocular toxoplasmosis at the age of 7, with two relapses of the disease in the past. The best-corrected visual acuity (BCVA) in both eyes was 1,0 on Snellen charts. The intraocular pressure was significantly increased up to $40 \mathrm{mmHg}$ in the right eye and $26 \mathrm{mmHg}$ in the left eye. Fundus examination revealed vitritis and the active whitish foci of choroidoretinitis at the macula and the presence of an old choroidoretinal scar below the optic nerve head in the right eye. In the left eye, an inactive choroidoretinal scar localized at the macula was discovered (Fig. 1 and Fig. 2). We conducted an optical coherence tomography (OCT) scan, however, the right eye scan was non-diagnostic due to

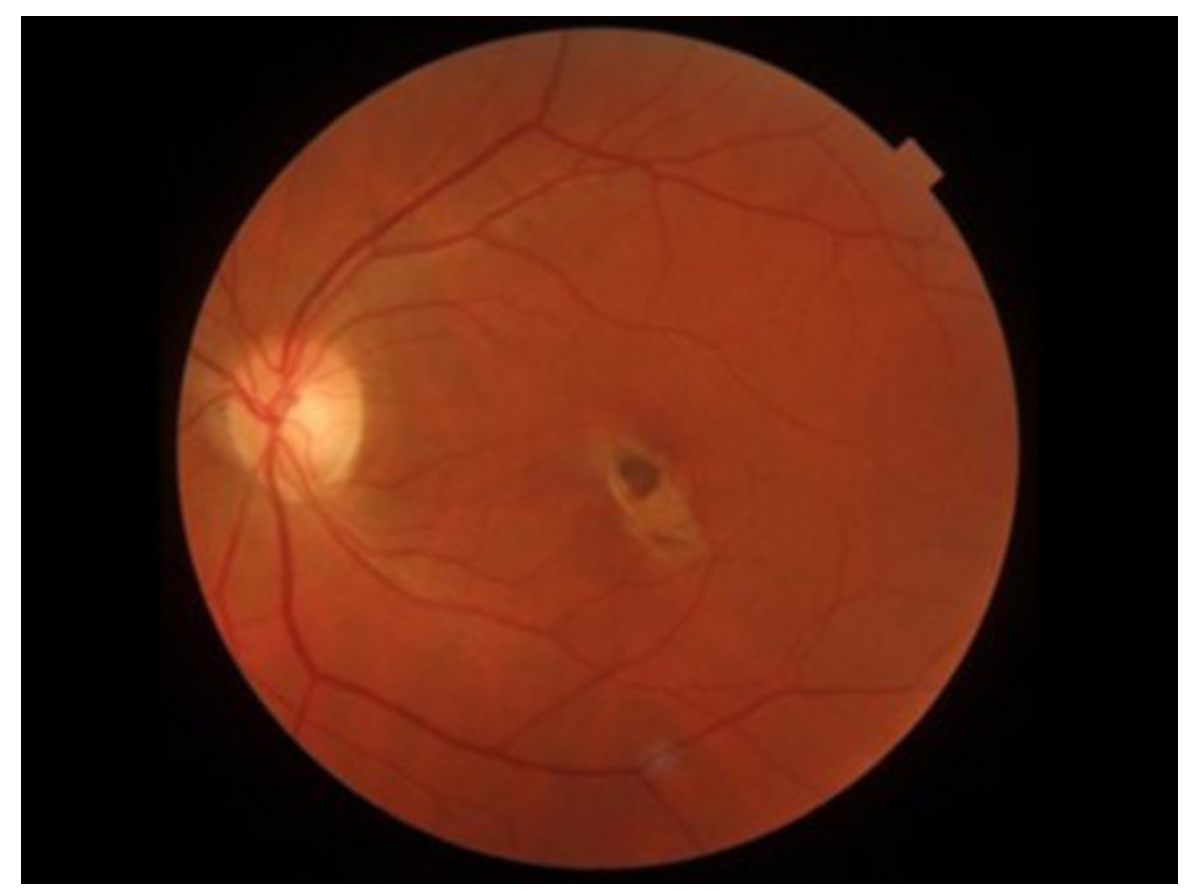

FIGURE 1 On admission, color fundus photograph of the left eye showing an inactive retinochoroidal scar
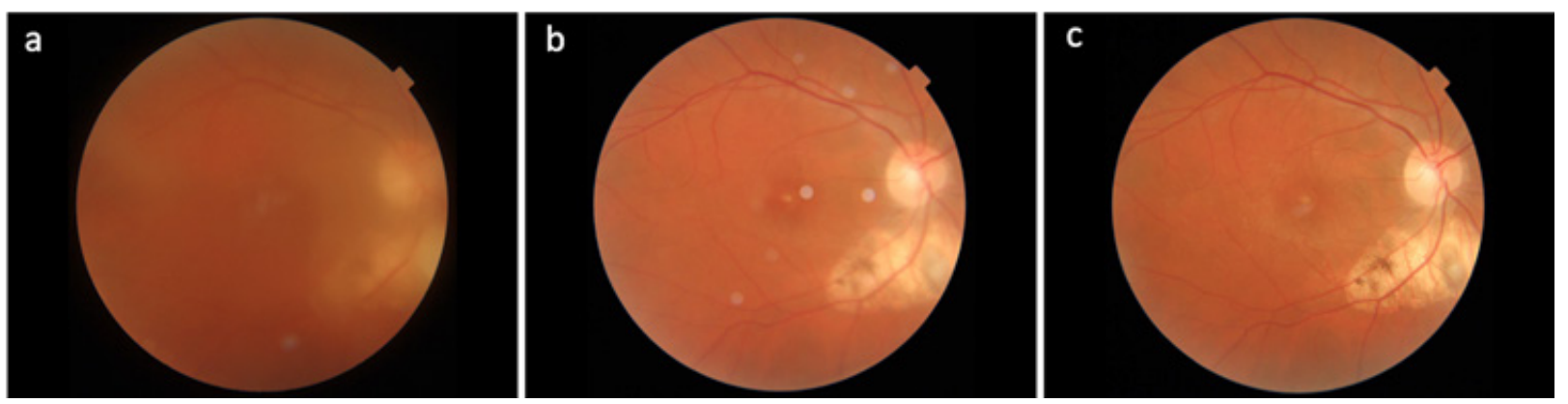

\section{FIGURE 2}

a - Blurred color fundus photograph of the right eye due to severe vitritis

b,c - Follow up, color fundus photograph of the right eye showing resolution of the lesions 


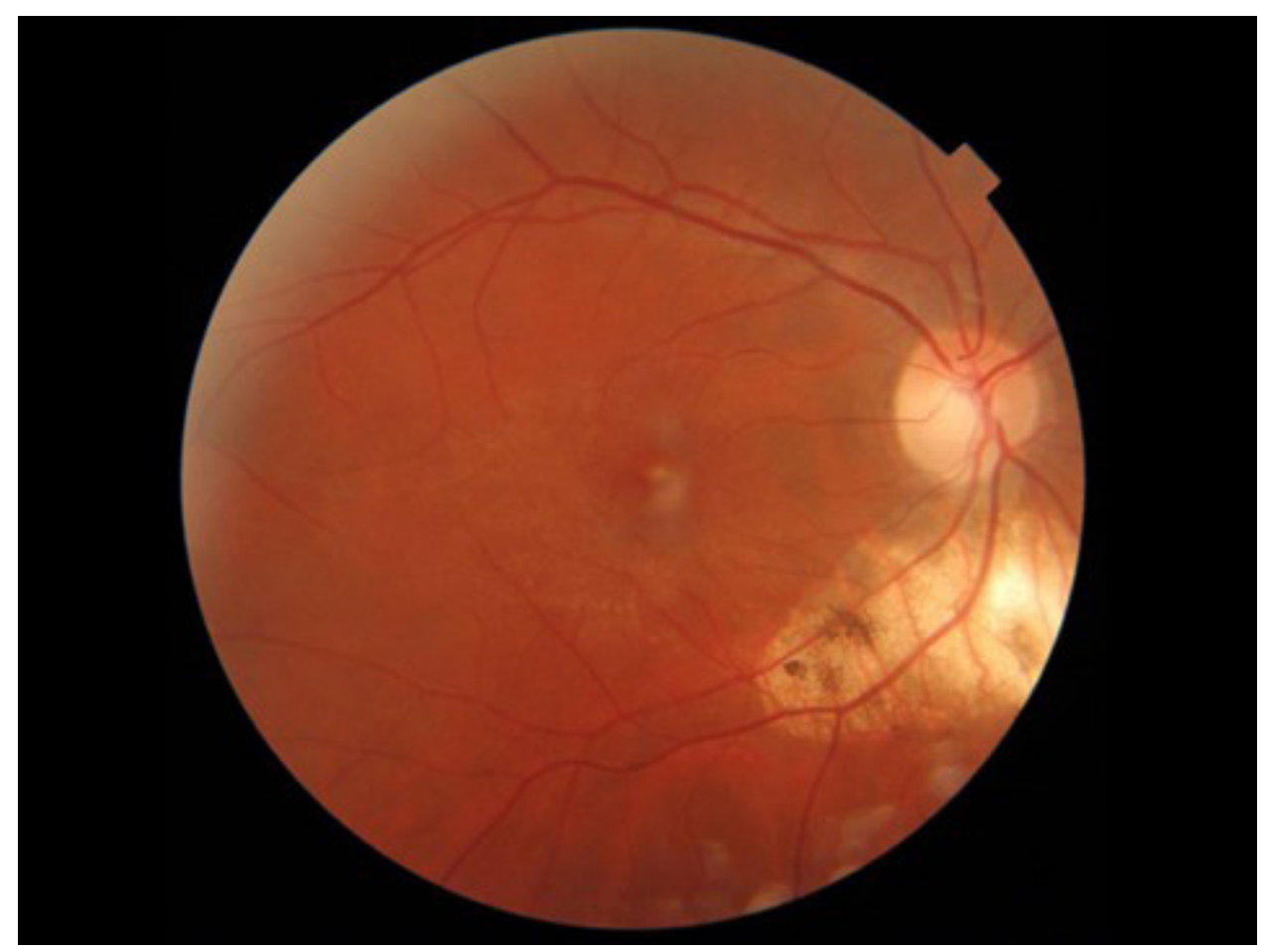

FIGURE 3 Photograph of the fundus of the right eye presenting resolution of ocular toxoplasmosis following the treatment

inflammation of the vitreous body. The examination suggested a relapse of congenital toxoplasmosis. The patient received topical (dexamethasone) and oral (prednisone) steroids together intraocular pressure-lowering drugs - brimonidine, dorzolamide, and timolol. The patient was transferred to the Department of Infectious Diseases, Hepatology, and Acquired Immunodeficiencies in Poznan. The laboratory tests showed an increased level of IgG against Toxoplasma gondii with low IgM. Pyrimethamine with sulfadiazine and clindamycin were implemented and the treatment with dexamethasone and prednisone was maintained. However, after two weeks, the patient was consulted by an ophthalmologist due to significant deterioration of the vision in the right eye. The ultrasound scan showed multiple opacities in the vitreous body of the right eye; thus, the patient received a higher dosage of prednisone. After three weeks of treatment, the visual acuity improved, and we achieved partial remission of the disease (Fig. 3). However, two months after therapy, the patient developed corticonuclear cataract in both eyes and reported psychotic symptoms. Hence, we lowered the dose of corticosteroids with a good clinical effect. The patient stays under control of our Outpatient Clinic preparing for cataract surgery.

\section{Discussion}

Ocular toxoplasmosis is one of the most common causes of infectious posterior uveitis [3]. It might be either congenital or acquired. Congenital ocular toxoplasmosis is asymptomatic in most infants. Nevertheless, a lot of untreated infants develop ocular symptoms in adulthood, which might result in severe visual impairment [4]. Typically, ocular toxoplasmosis presents as retinochoroiditis, with lesions identified as focal necrotizing retinitis. Active lesions appear as whitish foci of retinochoroiditis and are usually found next to atrophic scars. Scars appear after the healing of active lesions. Another common symptom of ocular toxoplasmosis is a severe vitritis, which appears as a "headlight in the fog" (a bright, white reflex in the eye, seen in an ophthalmoscope) [5]. Sometimes ocular toxoplasmosis might lead to cataract formation, retinal detachment, and optic nerve atrophy. Ocular manifestations of toxoplasmosis frequently recur, usually at school-entry age and during adolescence [4]. On examination, it is impossible to distinguish between the recurrence of congenital infection and postnatally acquired infection, serologic tests are useful to determine that [6]. In patients with active retinochoroiditis (due to reactivation of congenital/ past infection), the low titres of IgG are present, but IgM are undetectable. In patients with postnatal or primary infection IgG and IgM titres are elevated. Moreover, a specific IgG antibody avidity test may be carried out to distinguish between active (low avidity index) and previous infection (high avidity index) [7]. A thorough medical interview is helpful to suspect or exclude congenital toxoplasmosis.

Some risk factors increase the chance of acquiring toxoplasmosis in pregnancy, for example, possession of a domestic cat or eating undercooked, raw meat [8]. Our patient was diagnosed with ocular toxoplasmosis when she was 7 years old, however, doctors determined it due to focal necrotizing retinitis, the avidity test was not performed. As a 
newborn, she did not have a screening for toxoplasmosis. However, numerous relapses of the disease throughout her life, the antibody profile, and the fundus scar suggest vertical transmission. In the majority of the cases, retinal lesions limit themselves. Nonetheless, the pathological changes may lead to permanent vision loss, as a consequence of glaucoma, cataracts, retinal detachment, etc.[4]. Antibiotics and corticosteroids do not eliminate the presence of the parasite, but they reduce the size of retinochoroidal scar, the possibility of recurrence, and the length of the acute infectious period [9]. The classic treatment is "triple-drug therapy", which consists of pyrimethamine, sulfadiazine, and corticosteroids. Combinations of trimethoprim and sulfamethoxazole are effective alternatives for sulfadiazine and pyrimethamine [3]. It is important to remember that trimethoprim sulfamethoxazole, pyrimethamine, and sulfadiazine may be myelotoxic $[3,10]$. To prevent this adverse effect, the patient should supplement folinic acid during treatment $[10,11]$. Thus, laboratory tests including morphology, liver enzyme, and creatinine levels should also be performed in the first place[3]. Additionally, clindamycin can be combined with "triple therapy". It is administered orally and concentrates mostly in ocular tissues. Steroids cannot be applied in monotherapy, since they may cause large retinal lesions [3,12]. As a consequence, they are usually added to the therapy to suppress the inflammation. Prednisone is a drug of choice, it is administered orally, but it might also be used in a topical form [3] The other local treatment option is the intravitreal injection of clindamycin and dexamethasone. This method provides a high intraocular concentration of the drugs and eliminates systemic side effects $[3,13]$. Our patient was treated with classic triple-drug therapy with the addition of clindamycin and topical steroids. We increased the dose of oral prednisone from $40 \mathrm{mg}$ to $70 \mathrm{mg}$, due to poor visual acuity and progression of the disease, as a result, the patient developed a corticonuclear cataract in both eyes and reported psychotic symptoms. Cataract could have been a result of the progression of toxoplasmosis or systemic corticosteroid therapy. One of the side effects of systemic steroids is psychiatric disturbances and this could have been a reason for psychotic symptoms in our patient [14]. Therefore, the clinical benefit of treatment should always be weighed against side effects for each patient.

\section{Conclusions}

In most cases, congenital ocular toxoplasmosis is primarily asymptomatic, however, delay in diagnosis and causal treatment may result in permanent visual loss. Toxoplasmosis should always be considered as a differential diagnosis in patients with uveitis. A thorough medical interview is important to identify patients with $\mathrm{CT}$, to schedule regular follow-ups, identify new lesions and provide treat- ment. It is crucial to manage every case individually and weigh the clinical benefit of treatment against side effects for each patient.

\section{Ethical approval}

The research related to human use has been complied with all the relevant national regulations, institutional policies and in accordance the tenets of the Helsinki Declaration. As all of the data presented were collected during routine clinical practice without any additional clinical procedures or inclusion of patient sensitive data, no Ethical Committee approval is needed for this study.

\section{Informed consent statement}

Informed consent has been obtained from all individuals included in this study.

\section{Acknowledgements}

Not applicable.

\section{Corresponding author}

Aleksandra Krasińska, Department of Ophthalmology, Poznań University of Medical Sciences, Poznań, Poland, email: alex.krasinska@gmail.com.

\section{Conflict of interest}

The authors declare they have no conflict of interest.

\section{References}

1. Maldonado YA, Read JS. Committee on Infectious Diseases. Diagnosis, Treatment, and Prevention of Congenital Toxoplasmosis in the United States. Pediatrics. 2017;139(2):e20163860; DOI:10.1542/ peds.2016-3860.

2. European Centre for Disease Prevention and Control. Congenital toxoplasmosis. In: ECDC. Annual epidemiological report for 2016. Stockholm:ECDC. 2019.

3. Ozgonul C, Besirli CG. Recent Developments in the Diagnosis and Treatment of Ocular Toxoplasmosis. Ophthalmic Res. 2017;57(1):1-12; DOI:10.1159/000449169.

4. McLeod R, Boyer K. Toxoplasmosis (Toxoplasma gondii). In: Kliegman RA, St Geme JW, Blum NJ, Shah SS, Tasker RC, Wilson KM , Behrman RE, editors. Nelson Textbook of Pediatrics. 21th ed. Philadelphia: Elsevier Health Sciences. 2019;316:1865-1877.

5. Park Y-H, Nam H-W. Clinical Features and Treatment of Ocular Toxoplasmosis. Korean J Parasitol. 2013;51(4):393-9; DOI:10.3347/ kjp.2013.51.4.393.

6. Muñoz-Roldan M, Heimesaat MM, Liesenfeld O. Toxoplasmosis. In Manson's Tropical Infectious Diseases. Elsevier. 2014;652-663; DOI:10.1016/B978-0-7020-5101-2.00049-2.

7. Giugno S, Monteleone MM, Insalaco G, Leanza G, Palumbo M, Leanza V. Congenital toxoplasmotic chorioretinitis following reinfection. Eur J Obstet Gynecol Reprod Biol. 2020; 251:263-265; DOI:10.1016/j. ejogrb.2020.04.045

8. Sáfadi MAP, Berezin EN, Farhat CK, Carvalho ES. Clinical presentation and follow up of children with congenital toxoplasmosis in Brazil. Braz J Infect Dis. 2003;7(5); DOI:10.1590/S1413-86702003000500007.

9. Feliciano-Alfonso JE, Vargas-Villanueva A, Marin MA, Trivino L, Carvajal N, Moreno M, Luna T, Lopez de Mesa C, Muñoz-Ortiz J, de-la-Torre A. Antibiotic treatment for ocular toxoplasmosis: a systematic review and meta-analysis: study protocol. Syst Reviews 8. 2019;146; DOI:10.1186/ s13643-019-1067-8.

10. Kaye A. Toxoplasmosis: Diagnosis, Treatment, and Prevention in Congenitally Exposed Infants. J Pediatr Health Care. 2011;25(6):355-64; DOI:10.1016/j.pedhc.2010.04.008.

11. Jones JL, Ogunmodede F, Scheftel J, Kirkland E, Lopez A, Schulkin J, Lynfield R. Toxoplasmosis-Related Knowledge and Practices Among Pregnant Women in the United States. Infect Dis Obstet Gynecol. 2003;11(3):139-45; DOI:10.1080/10647440300025512.

12. Oray M, Ozdal PC, Cebeci Z, Kir N, Tugal-Tutkun I. Fulminant Ocular Toxoplasmosis: The Hazards of Corticosteroid Monotherapy. Ocul Immunol Inflamm. 2016;24(6):637-646; DOI:10.3109/09273948.2015.1 057599.

13. Soheilian M, Ramezani A, Azimzadeh A, Sadoughi MM, Dehghan MH, Shahghadami R, Yaseri M, Peymanet GA. Randomized Trial of Intravitreal Clindamycin and Dexamethasone versus Pyrimethamine, Sulfadiazine, and Prednisolone in Treatment of Ocular Toxoplasmosis. Oph thalmology. 2011;118(1):134-41; DOI:10.1016/j.ophtha.2010.04.020.

14. Prednisone: Drug information [Internet]. UpToDate: Lexicomp; Copyright 1978-2021. Prednisone [cited 10 May 2021]. Available from: https://www.uptodate.com/contents/ prednisone-drug-information?topicRef $=850$ \&source $=$ see_link 